\title{
Adaptive Clutter Filter Design for Micro-Ultrasound Color Flow Imaging of Small Blood Vessels
}

\author{
Dave K. H. Cheung*, Harry C. T. Chiu*, Lequan Zhang ${ }^{\dagger}$, Changhong $\mathrm{Hu}^{\dagger}$, \\ K. Kirk Shung ${ }^{\dagger}$, and Alfred C. H. Yu* \\ * Medical Engineering Program, The University of Hong Kong, Pokfulam, Hong Kong SAR \\ ${ }^{\dagger}$ Ultrasonic Transducer Resource Center, The University of Southern California, USA \\ Corresponding Email: alfred.yu@hku.hk
}

\begin{abstract}
In micro-ultrasound, which uses imaging frequencies above $20 \mathrm{MHz}$, obtaining color flow images (CFI) of small blood vessels using is not a trivial task because it is more challenging to suppress tissue clutter properly given the stronger blood signal power at high imaging frequencies and the slow blood velocity inside the microcirculation. To improve clutter suppression in micro-ultrasound CFI, this paper presents an adaptive clutter filtering approach that is based on a two-stage eigen-analysis of slow-time ensemble characteristics. The approach first identifies tissue pixels in the imaging view by examining whether highfrequency contents are absent in the principal slow-time eigencomponents for each pixel as computed from single-ensemble eigen-decomposition. It then computes the filtered slow-time ensemble for each pixel by finding the least-squares projection residual between the pixel's slow-time ensemble and the clutter eigen-components estimated from a multi-ensemble eigendecomposition of tissue slow-time ensembles within a spatial window. In this filtering approach, the clutter eigen-components are chosen based on whether their mean frequency lies within a spectral band. To analyze the efficacy of the proposed adaptive filter, both in-vitro experiments and Field II simulations were carried out. For the experiments, raw CFI data were acquired using a 64-element, $33 \mathrm{MHz}$ linear array prototype (pulse duration: 2 cycles, PRF: $1 \mathrm{kHz}$, transmit focus: 8mm, F-number: 5). Their imaging view corresponded to the cross-section of a $0.9 \mathrm{~mm}$-diameter tube that was placed on top of an unsuspended table where ambient vibrations may appear; flow velocity $(5,7$, $10,15 \mathrm{~mm} / \mathrm{s}$ ) within the tube was controlled using a syringe pump. For the simulations, raw CFI data was computed for both plug and parabolic flow profiles, and tissue motion was modeled as $0.5 \mathrm{~mm} / \mathrm{s}$ sinusoidal vibrations. For all flow velocities tested in our in-vitro study, the proposed adaptive filter improved the flow detection sensitivity as compared to existing ones. In the slowflow case $(5 \mathrm{~mm} / \mathrm{s})$, we observed over $70 \%$ increase in flow detection sensitivity (assuming a $5 \%$ false alarm rate). This effectively reduced flashing artifacts in the resulting CFIs and gave a more consistent visualization of the flow tube.
\end{abstract}

Keywords-micro-ultrasound, color flow imaging, clutter filter, flow detection, microcirculation.

\section{INTRODUCTION}

Microcirculation is known to play an important role in our bodies. It is responsible for the exchange of nutrients and metabolic wastes in the surrounding tissue. The study of the microcirculation inside these small capillaries (together with the arterioles and venules) can provide valuable diagnostic and physiological information. For example, from physiology it is known that the angiogenesis (formation of new blood vessels) of capillaries can be triggered by wound healing process or cancer stimulation. In addition, small animal models like mice and rabbits are often employed to study various diseases and the flow information inside their small vessels is often wanted. Micro-ultrasound with frequency greater than $20 \mathrm{MHz}$ can be one of the imaging modalities to image the blood flow inside these small vessels. Several animal studies have been performed using micro-ultrasound to measure blood flow information, such as detecting for angiogenesis in the vicinity of mouse tumors [1] and assessing the recovery of damaged mouse Achilles tendons through identifying newly generated micro-circulation [2]. These studies show that micro-ultrasound color flow imaging (CFI) can possibly develop itself into a useful tool for clinical diagnoses.

Although micro-ultrasound CFI has potential in several clinical applications, its feasibility is highly dependent on the sensitivity and specificity in detecting blood flow regions. This in turn depends on the clutter filtering performance during the slow-time signal processing. In general, there are three unique issues relevant to clutter filtering in micro-ultrasound CFI: 1) high blood-to-clutter signal power ratio (BCR), 2) slow flow velocities in the microcirculation, and 3) signal decorrelation if data is acquired using a swept-scan approach. While the third challenge can be addressed by the recent development of highfrequency linear arrays [3], the other two challenges can significant affect the effectiveness of existing clutter filters when they are applied to the micro-ultrasound scenarios.

In this work, we present a new adaptive clutter filter that is designed to address the clutter suppression challenges in microultrasound CFI. The theoretical principles of our proposed filter will be explained in the next section. Results from simulation studies and flow phantom experiments will then be discussed to illustrate the proposed filter's performance.

\section{THEORY}

\section{A. Overall Description}

Fig. 1 shows a block diagram illustration of the proposed adaptive clutter filtering approach. It essentially consists of two processing stages. First, it identifies tissue pixels in the imaging view by performing a single-ensemble eigen-analysis on the slow-time ensemble of each pixel position and identifying tissue pixels as ones whose principal eigencomponents do not bear high-frequency contents that are 
Stage 1: Identifying Tissue Pixels

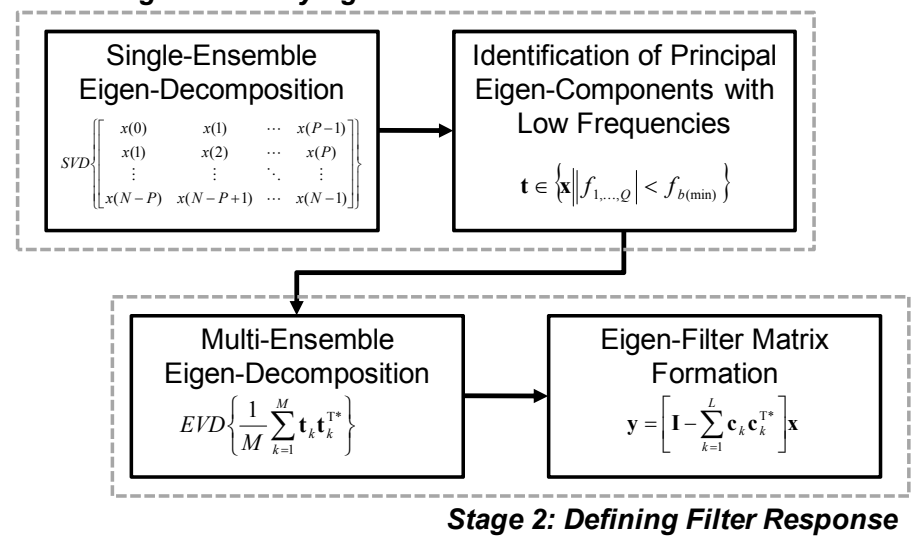

Fig. 1. Block diagram illustration of the processing stages involved in the proposed eigen-based filtering approach for micro-ultrasound CFI.

presumably brought about by blood flow. Second, it calculates the filtered slow-time ensemble for each pixel by carrying out multi-ensemble eigen-filtering based on the slow-time signal statistics of the identified tissue pixels within a spatial window centered locally around the pixel of interest. The following subsections provide a more detailed discussion on the formulation of each processing stage.

\section{B. Stage 1: Identifying Tissue Pixels}

From an eigen-analysis perspective, the clutter filtering problem in micro-ultrasound CFI is different from the one in conventional CFI because the fine spatial resolution at high imaging frequencies inherently implies that the slow-time ensembles of adjacent tissue pixels are likely to be statistically stationary. As such, it seems practical to adaptively define the filter response of each pixel through a multi-ensemble eigenestimation formulation that can more effectively represent clutter using eigen-components with arbitrary waveforms [4]. Nevertheless, because of the high BCR under microultrasound imaging settings, it is essential to exclude slowtime ensembles of blood regions from the multi-ensemble eigen-estimation process in order to avoid concomitant suppression of blood signals by the eigen-based filter. Proper identification of tissue pixels in the imaging view is thus a necessary first step in developing an eigen-filter for microultrasound CFI.

In this work, tissue pixels are identified by performing a single-ensemble eigen-analysis on each slow-time ensemble. Such a strategy is essentially based on an analysis of every slow-time ensemble's principal signal contents that are computed through a Hankel singular value decomposition (Hankel-SVD) approach satisfying the following relation:

$$
\mathbf{H}=\left[\begin{array}{cccc}
x(0) & x(1) & \cdots & x(P-1) \\
x(1) & x(2) & \cdots & x(P) \\
\vdots & \vdots & \ddots & \vdots \\
x(N-P) & x(N-P+1) & \cdots & x(N-1)
\end{array}\right]=\sum_{k=1}^{P} \sigma_{k} \mathbf{u}_{k} \mathbf{v}_{k}^{\mathrm{T}^{*}} .
$$

In the above, $x(n)$ is the $n^{\text {th }}$ sample in a size- $N$ slow-time ensemble, $P$ is the number of eigen-components in the single- ensemble eigen-estimation formulation (at most $N / 2$ ), $\sigma_{k}$ is the $k^{\text {th }}$ singular value, and $\mathbf{u}_{\boldsymbol{k}}, \mathbf{v}_{\boldsymbol{k}}$ are the corresponding left and right singular vectors computed from the SVD of Hankel data matrix $\mathbf{H}$. The primary advantage of using a single-ensemble eigen-estimation approach in this processing stage is that the principal slow-time signal contents may be computed individually for each pixel independent of the slow-time signal characteristics of neighboring pixels (as required for multiensemble eigen-estimation) [4]. This helps to ensure that the eigen-analysis for tissue pixel identification is carried out under spatially independent conditions.

Based on the slow-time eigen-components computed (in the form of singular vectors $\mathbf{u}_{k}$ or $\mathbf{v}_{k}$ ), tissue pixels may be identified through an analysis of the components' mean frequencies that can be found from the lag-one autocorrelator [5]. Given that blood flow is generally faster than the tissue motion, we have classified a pixel to be within the tissue region if none of its principal eigen-components have a mean frequency higher than a pre-defined frequency threshold. In other words, a tissue slow-time ensemble $\mathbf{t}$ is defined based on the following rule:

$$
\mathbf{t} \in\left\{\mathbf{x}|| f_{1, \ldots, Q} \mid<f_{b(\min )}\right\},
$$

where $\mathbf{x}$ denotes a slow-time ensemble vector, $f_{1, \ldots, Q}$ denotes the mean frequencies of the $Q$ largest eigen-components of $\mathbf{x}$ as computed from the SVD of (1), and $f_{b(\text { min })}$ denotes the minimum Doppler frequency of blood flow. Note that only the principal components are considered in our classification criterion in order to exclude weak noise components that may have spurious high frequency. Also, the frequency threshold $f_{b(\min )}$ may be set according to the target minimum imaging flow velocity which can be transformed into a frequency value through the Doppler equation.

\section{Stage 2: Defining Filter Response}

After the tissue pixels have been identified, their slow-time ensembles are used to adaptively form the filter response at different pixel positions. In this processing stage, the multiensemble eigen-estimation approach is used so that the resulting filter attenuation characteristics may be more effective as clutter may be epitomized using arbitrary-shaped eigen-components [4].

The filter response for each pixel is defined by first computing the clutter eigen-components based on tissue slowtime ensembles over a spatial window centered around that pixel. This process can essentially be regarded as a three-step procedure. First, a slow-time correlation matrix $\mathbf{R}$ is formed for a pixel position through averaging the outer product of each tissue slow-time ensemble (within the spatial window) and its conjugate transpose:

$$
\mathbf{R}=\frac{1}{M} \sum_{k=1}^{M} \mathbf{t}_{k} \mathbf{t}_{k}^{\mathrm{T}^{*},}
$$

where $\mathbf{t}_{k}$ denotes one of the $M$ tissue slow-time ensembles within the spatial window. Subsequently, the eigendecomposition of $\mathbf{R}$ is computed to find the set of eigenvalues $\lambda_{k}$ and eigenvectors $\mathbf{e}_{k}$ that satisfy the following relation: 
TABLE I. IMAging PARAmeters For the Performance AsSESSMent

\begin{tabular}{cc}
\hline Parameter & Value \\
\hline Center Frequency & $33 \mathrm{MHz}$ \\
RF Sampling Frequency & $200 \mathrm{MHz}$ \\
Pulse Repetition Frequency & $1 \mathrm{kHz}$ \\
Array Size & 64 Elements \\
Aperture Size & 32 Eleemnts \\
Pulse Duration & 2 Cycles \\
Slow-Time Ensemble Size & 16 \\
\hline
\end{tabular}

$$
\mathbf{R}=\sum_{k=1}^{N} \lambda_{k} \mathbf{e}_{k} \mathbf{e}_{k}^{\mathrm{T} *} .
$$

After that, the clutter eigen-components are identified through finding the low-frequency eigenvectors whose frequency can be readily estimated from the lag-one autocorrelator [5]. This step can be regarded as selecting the order of the adaptive clutter filter for a pixel through a frequency-thresholding algorithm, which is known to be more robust than eigenvalue thresholding [4].

With the identified clutter eigen-components for a pixel, filtering is performed through a least-squares projection that computes the fitting residual between the clutter eigenvectors and the slow-time ensemble for that pixel. As such, the filtered slow-time ensemble y is mathematically given by:

$$
\mathbf{y}=\left[\mathbf{I}-\sum_{k=1}^{L} \mathbf{c}_{k} \mathbf{c}_{k}^{\mathrm{T}^{*}}\right] \mathbf{x},
$$

where $\mathbf{I}$ is an $N \times N$ identity matrix, and $\mathbf{c}_{k}$ represents one of the $L$ clutter eigen-components in the set. It is worth emphasizing that this filter formulation is applied separately to each pixel in the imaging view. Hence, the filter is adaptive to the local tissue slow-time ensemble characteristics.

\section{Performance Assessment Methods}

\section{A. Simulation Study}

Field-II simulation studies were conducted to assess the proposed filter's performance and to compare it with a few different existing clutter filters (polynomial regression filter, clutter-downmixing filter). Both plug flow (for $0.4 \mathrm{~mm}$ tubes positioned at two different depths $(7.7 \mathrm{~mm}, 8.3 \mathrm{~mm})$ with 1$10 \mathrm{~mm} / \mathrm{s}$ flow) and parabolic flow (for $0.7 \mathrm{~mm}$ tube centered at $8 \mathrm{~mm}$ depth with $0-10 \mathrm{~mm} / \mathrm{s}$ flow) were considered, and tissue motion was modeled as a $3 \mathrm{~Hz}, 0.5 \mathrm{~mm} / \mathrm{s}$ sinusoidal vibration. Raw CFI data (30 sets for each flow velocity) was synthesized for an imaging setup involving a 64-element, $33 \mathrm{MHz}$ lineararray probe (transmit focus: $8 \mathrm{~mm}$, F-number: 5). The main imaging parameters are summarized in Table I.

\section{B. Flow Phantom Experiments}

To evaluate the filtering performance in practical situations, an in-vitro wall-less flow phantom imaging experiment was performed as illustrated in Fig. 2. The imaging system used in this work [6] had the same specifications as the ones used in the simulations (see Table I). The 0.9mm-diameter wall-less tube phantom was positioned at $8 \mathrm{~mm}$ deep within a tissuemimicking material [composition ( $\%$ by weight): $87.8 \%$ DI water, $8.1 \%$ 1-propanol, $0.2 \%$ formaldehyde, $1.9 \%$ agar and $2.0 \% \mathrm{SiO}_{2}(0.5-10 \mu \mathrm{m}$ diameter $\left.)\right]$, and it had a measured BCR of $-7.24 \pm 0.42 \mathrm{~dB}$. In the experiments, the phantom was placed

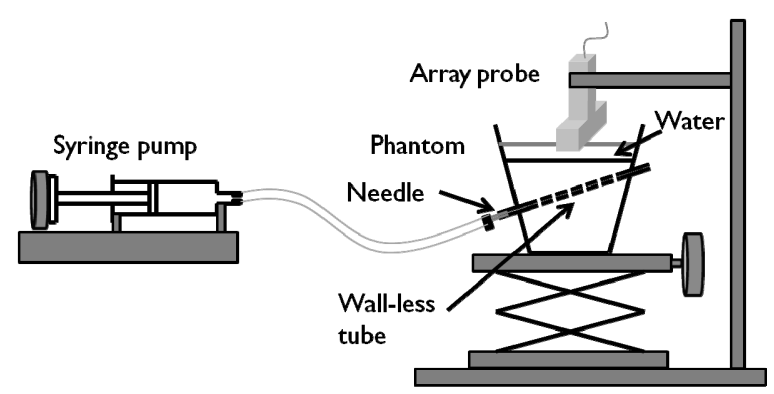

Fig. 2. In-vitro setup used for experimental evaluation of the proposed eigenbased filtering approach for micro-ultrasound CFI.
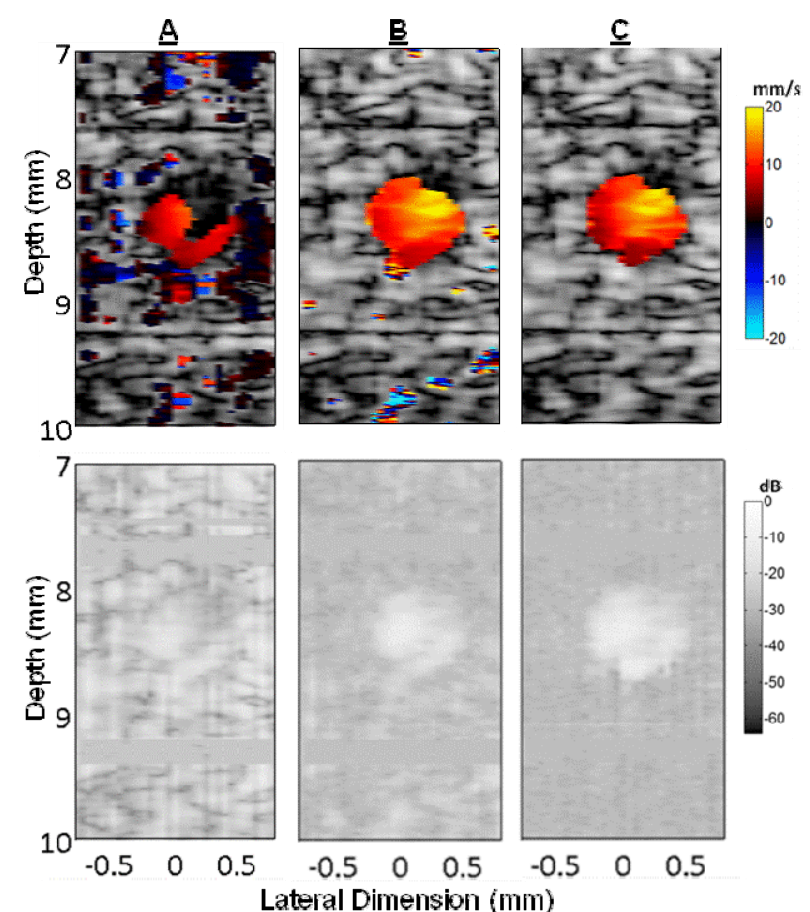

Fig. 3. CFIs and Doppler power maps produced at flow velocity of $10 \mathrm{~mm} / \mathrm{s}$ in the flow-phantom experiment using different clutter filters: a) polynomial regression, b) clutter downmixing, and c) proposed filter.

on top of an unsuspended table where ambient vibrations may appear. Flow velocity $(5,7,10,15 \mathrm{~mm} / \mathrm{s})$ within the tube was controlled using a syringe pump.

\section{Analysis Proedure}

To analyze the clutter rejection performance of the filters, the receiver operating characteristics of the filters were evaluated at different flow velocities for a fixed false alarm rate $(5 \%$ in this study). In addition, the estimated flow profiles of the parabolic flow simulation model after clutter filtering were compared against the ideal flow profile to compute the estimation bias and variance so as to decide which filter could provide a better resemblance to the ideal flow profile.

\section{RESUlts AND Discussion}

\section{A. CFIs and Doppler Power Maps}

Fig. 3 shows the CFIs and Doppler power maps produced with different clutter filters for the in-vitro experiment at $10 \mathrm{~mm} / \mathrm{s}$ flow velocity. It can be seen from the Doppler power maps that the proposed filter (Fig. $3 \mathrm{c}$ ) provided the best clutter 

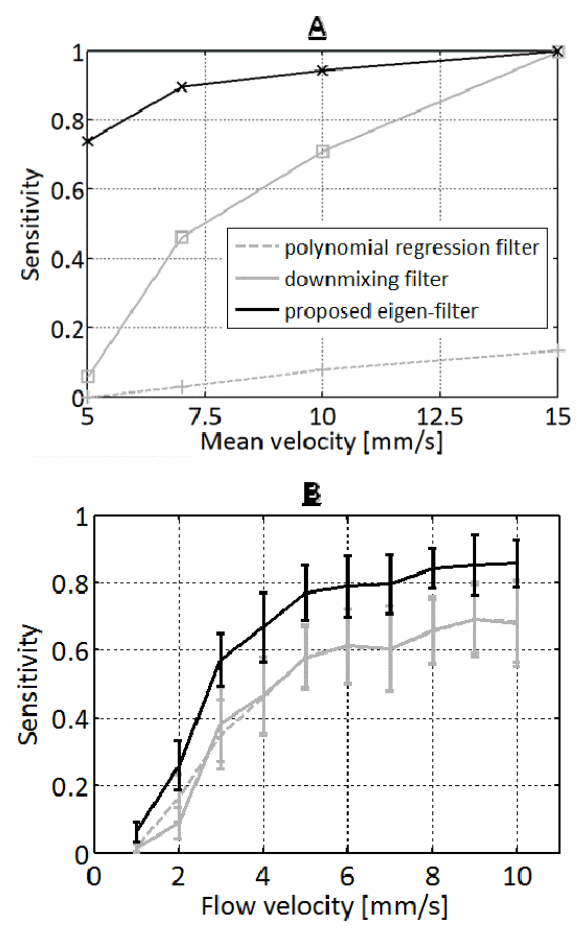

Fig. 4. Flow detection sensitivity at different flow velocities for a $5 \%$ false alarm rate: a) in-vitro experiment, b) simulation study with -20dB BCR.

suppression as compared with other filters, leading to a clear identification of the flow region in the resultant CFI. This should be contrasted with the results for the polynomial regression filter (Fig. 3a) and clutter-downmixing filter (Fig. $3 b$ ), where it can be seen that the clutter power was not suppressed adequately. Similar result trends were observed for other flow velocities and in the simulation analysis.

\section{B. Flow Detection Performance}

Fig. 4a shows the sensitivity (\% of correctly identified blood region) of different filters at false alarm rate of 0.05 (i.e. $5 \%$ of tissue region being wrongly identified as blood region) at different flow velocities in the in-vitro study. As can be seen, at all flow velocities, the proposed filter improved the flow detection sensitivity as compared to the other two filters. In the slow-flow case $(5 \mathrm{~mm} / \mathrm{s})$ in the in-vitro study, there was over $70 \%$ increase in flow detection sensitivity. This effectively reduced flashing artifacts in the resulting CFIs and gave a more consistent visualization of the flow tube.

Fig. $4 \mathrm{~b}$ shows a similar plot for the plug flow simulation model at $-20 \mathrm{~dB}$ BCR. It can be observed that the proposed filter provided the best flow detection sensitivity among all filters. For the OdB BCR scenario (not shown here), owing to the higher blood signal power, the flow detection sensitivities of the proposed filter and other filters were similar.

\section{Bias and Variance Analysis}

Fig. 5 shows the estimation bias and variance for different filters in the parabolic flow simulation model at BCR of $0 \mathrm{~dB}$. An over-estimation of the flow velocity in the slow blood flow regions $(<5 \mathrm{~mm} / \mathrm{s})$ were observed in all of the filters, owing to the removal of slow blood flow signals. However, comparing to other filters, the proposed filter gave the smallest estimation
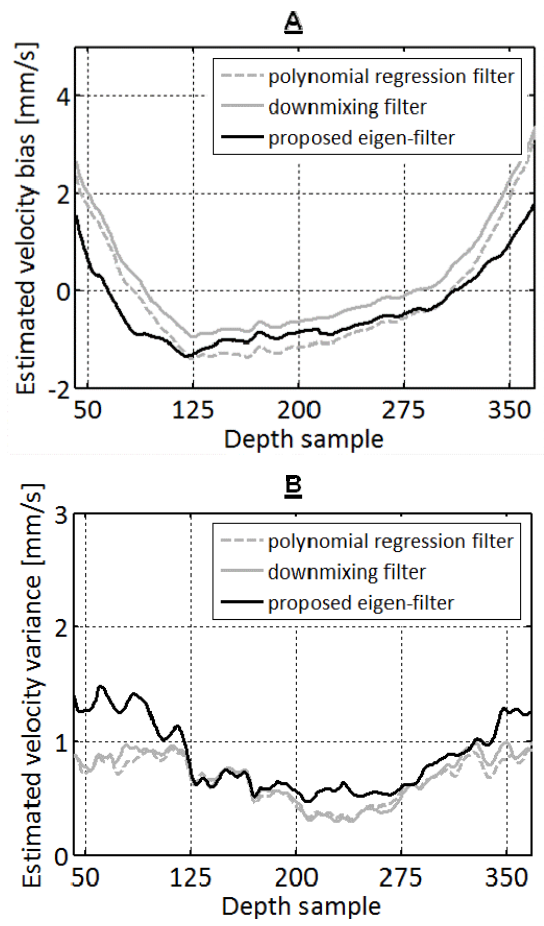

Fig. 5. Estimation anaylsis for a simulation study conducted on a parabolic flow tube with $10 \mathrm{~mm} / \mathrm{s}$ center-line velocity. Results are shown for different clutter filters in a case with OdB BCR: (a) velocity bias and (b) variance.

bias in slow flow region (around $1 \mathrm{~mm} / \mathrm{s}$ lower). A better resemblance to the actual flow profile could thus be achieved which could be useful when an analysis of flow profile was required in research or clinical applications. Although it seems that the estimation variance of the proposed filter is a slightly higher at the boundary region, its effect on the flow profile is less significant than that of the estimation bias since it can be lowered by data averaging (whereas for the estimation bias, this cannot be done). A similar trend was observed in the case of $-20 \mathrm{~dB} B C R$.

\section{ACKNOLWEDGEMENTS}

We are very grateful to Prof. Paul Cheung at HKU for his enthusiastic support on this work.

\section{REFERENCES}

[1] F. S. Foster, "Micro-ultrasound takes off (In the biological sciences)," in Proc. IEEE Ultrason. Symp., pp. 120-125, 2008.

[2] C. K. Yeh, J. J. Chen, M. L. Li, and J. J. Luh, "In vivo imaging of blood flow in the mouse Achilles tendon using high-frequency ultrasound," Ultrasonics, vol. 49, pp. 226-230, 2009.

[3] J. M. Cannata, J. A. Williams, Q. Zhou, T. A. Ritter, and K. K. Shung, "Development of a 35-MHz piezo-composite ultrasound array for medical imaging," IEEE Trans. Ultrason. Ferroelec. Freq. Contr., vol. 53, pp. 224-236, 2006.

[4] A. C. H. Yu and L. Lovstakken, "Eigen-based clutter filter design for ultrasound color flow imaging: a review," IEEE Trans. Ultrason. Ferroelec. Freq. Contr., vol. 57, pp. 1096-1111, 2010.

[5] C. Kasai, K. Namekawa, A. Koyano, and R. Omoto, "Real-time twodimensional blood flow imaging using an autocorrelation technique," IEEE Trans. Son. Ultrason., vol. 32, pp. 458-464, 1985.

[6] L. Zhang, X. Xu, C. Hu, L. Sun, J. T. Yen, J. M. Cannata, and K. K. Shung, "A high-frequency high frame rate duplex ultrasound linear array imaging system for small animal imaging", IEEE Trans. Ultrason. Ferroelec. Freq. Contr., vol. 57, pp. 1548-1557, 2010. 